\title{
Anhang 2c: Retweet-Netzwerk (Alternative Nachrichtenmedien)
}

\begin{tabular}{|c|c|c|c|c|c|c|c|}
\hline Account & $\begin{array}{c}\text { Modularity } \\
\text { Class }\end{array}$ & $\begin{array}{c}\text { In- } \\
\text { degree }\end{array}$ & $\begin{array}{c}\text { Out- } \\
\text { degree }\end{array}$ & Degree & $\begin{array}{c}\text { Close- } \\
\text { ness } \\
\text { Centrality } \\
\end{array}$ & $\begin{array}{l}\text { Betweenness } \\
\text { Centrality }\end{array}$ & $\begin{array}{l}\text { Eigen. } \\
\text { Centr. }\end{array}$ \\
\hline Volksverpetzer & $\begin{array}{l}\text { Deutsche } \\
\text { Alternativ- } \\
\text { medien Typ } \\
\text { III, IV }\end{array}$ & 6207 & 18 & 6225 & 0,084 & 27891640,946 & 1,000 \\
\hline BILDblog & $\begin{array}{l}\text { Deutsche } \\
\text { Alternativ- } \\
\text { medien Typ } \\
\text { III, IV }\end{array}$ & 2704 & 2 & 2706 & 0,478 & 63678,467 & 0,451 \\
\hline uebermedien & $\begin{array}{l}\text { Deutsche } \\
\text { Alternativ- } \\
\text { medien Typ } \\
\text { III, IV }\end{array}$ & 1546 & 8 & 1554 & 0,647 & 181306,064 & 0,278 \\
\hline lobbycontrol & $\begin{array}{l}\text { Deutsche } \\
\text { Alternativ- } \\
\text { medien Typ } \\
\text { III, IV }\end{array}$ & 886 & 3 & 889 & 1,000 & 28993,663 & 0,143 \\
\hline derfreitag & $\begin{array}{l}\text { Deutsche } \\
\text { Alternativ- } \\
\text { medien Typ } \\
\text { III, IV }\end{array}$ & 310 & 0 & 310 & 0,000 & 0,000 & 0,070 \\
\hline krautreporter & $\begin{array}{l}\text { Deutsche } \\
\text { Alternativ- } \\
\text { medien Typ } \\
\text { III, IV }\end{array}$ & 304 & 58 & 362 & 0,073 & 2441917,728 & 0,050 \\
\hline MartinSonneborn & $\begin{array}{l}\text { Deutsche } \\
\text { Alternativ- } \\
\text { medien Typ } \\
\text { III, IV }\end{array}$ & 281 & 0 & 281 & 0,000 & 0,000 & 0,044 \\
\hline Die_Insider & $\begin{array}{l}\text { Deutsche } \\
\text { Alternativ- } \\
\text { medien Typ } \\
\text { III, IV }\end{array}$ & 265 & 5 & 270 & 0,077 & 387936,562 & 0,058 \\
\hline
\end{tabular}




\begin{tabular}{|c|c|c|c|c|c|c|c|}
\hline Account & $\begin{array}{l}\text { Modularity } \\
\text { Class }\end{array}$ & $\begin{array}{c}\text { In- } \\
\text { degree }\end{array}$ & $\begin{array}{c}\text { Out- } \\
\text { degree }\end{array}$ & Degree & $\begin{array}{c}\text { Close- } \\
\text { ness } \\
\text { Centrality }\end{array}$ & $\begin{array}{c}\text { Betweenness } \\
\text { Centrality }\end{array}$ & $\begin{array}{l}\text { Eigen. } \\
\text { Centr. }\end{array}$ \\
\hline ARD_Presseclub & $\begin{array}{l}\text { Deutsche } \\
\text { Alternativ- } \\
\text { medien Typ } \\
\text { III, IV }\end{array}$ & 216 & 0 & 216 & 0,000 & 0,000 & 0,040 \\
\hline Kontrast_at & $\begin{array}{l}\text { Österreichi- } \\
\text { sche Alter- } \\
\text { nativmedien } \\
\text { Typ III, IV }\end{array}$ & 1032 & 1 & 1033 & 0,000 & 0,000 & 0,167 \\
\hline daswasfehlt & $\begin{array}{l}\text { Österreichi- } \\
\text { sche Alter- } \\
\text { nativmedien } \\
\text { Typ III, IV }\end{array}$ & 150 & 9 & 159 & 0,217 & 4504541,685 & 0,024 \\
\hline erstaunlich_at & $\begin{array}{l}\text { Österreichi- } \\
\text { sche Alter- } \\
\text { nativmedien } \\
\text { Typ III, IV }\end{array}$ & 52 & 0 & 52 & 0,000 & 0,000 & 0,009 \\
\hline misik & $\begin{array}{l}\text { Österreichi- } \\
\text { sche Alter- } \\
\text { nativmedien } \\
\text { Typ III, IV }\end{array}$ & 25 & 1 & 26 & 1,000 & 19111,000 & 0,004 \\
\hline ArminWolf & $\begin{array}{l}\text { Österreichi- } \\
\text { sche Alter- } \\
\text { nativmedien } \\
\text { Typ III, IV }\end{array}$ & 21 & 1 & 22 & 0,091 & 55572,334 & 0,003 \\
\hline RepublikMagazin & $\begin{array}{l}\text { Schweizer } \\
\text { Alternativ- } \\
\text { medien Typ } \\
\text { III, IV }\end{array}$ & 213 & 96 & 309 & 0,091 & 5272394,542 & 0,036 \\
\hline Knutti_ETH & $\begin{array}{l}\text { Schweizer } \\
\text { Alternativ- } \\
\text { medien Typ } \\
\text { III, IV }\end{array}$ & 213 & 7 & 220 & 0,084 & 2230485,564 & 0,035 \\
\hline JolandaSpiess & $\begin{array}{l}\text { Schweizer } \\
\text { Alternativ- } \\
\text { medien Typ } \\
\text { III, IV }\end{array}$ & 74 & 12 & 86 & 0,084 & 456361,260 & 0,012 \\
\hline elia_bluelle & $\begin{array}{l}\text { Schweizer } \\
\text { Alternativ- } \\
\text { medien Typ } \\
\text { III, IV }\end{array}$ & 51 & 18 & 69 & 0,084 & 173458,369 & 0,009 \\
\hline dossier_ & $\begin{array}{l}\text { Schweizer } \\
\text { Alternativ- } \\
\text { medien Typ } \\
\text { III, IV }\end{array}$ & 40 & 8 & 48 & 0,126 & 3010656,592 & 0,006 \\
\hline adfichter & $\begin{array}{l}\text { Schweizer } \\
\text { Alternativ- } \\
\text { medien Typ } \\
\text { III, IV }\end{array}$ & 29 & 16 & 45 & 0,084 & 116132,585 & 0,005 \\
\hline
\end{tabular}




\begin{tabular}{|c|c|c|c|c|c|c|c|}
\hline Account & $\begin{array}{l}\text { Modularity } \\
\text { Class }\end{array}$ & $\begin{array}{c}\text { In- } \\
\text { degree }\end{array}$ & $\begin{array}{c}\text { Out- } \\
\text { degree }\end{array}$ & Degree & $\begin{array}{l}\text { Close- } \\
\text { ness } \\
\text { Centrality }\end{array}$ & $\begin{array}{l}\text { Betweenness } \\
\text { Centrality }\end{array}$ & $\begin{array}{l}\text { Eigen. } \\
\text { Centr. }\end{array}$ \\
\hline ZwitscherLAMM & $\begin{array}{l}\text { Schweizer } \\
\text { Alternativ- } \\
\text { medien Typ } \\
\text { III, IV }\end{array}$ & 16 & 1 & 17 & 1,000 & 63,879 & 0,003 \\
\hline KlimaschutzCH & $\begin{array}{l}\text { Schweizer } \\
\text { Alternativ- } \\
\text { medien Typ } \\
\text { III, IV }\end{array}$ & 9 & 0 & 9 & 0,000 & 0,000 & 0,001 \\
\hline Lobbywatch_CH & $\begin{array}{l}\text { Schweizer } \\
\text { Alternativ- } \\
\text { medien Typ } \\
\text { III, IV }\end{array}$ & 9 & 0 & 9 & 0,000 & 0,000 & 0,002 \\
\hline WernerFuchs_CH & $\begin{array}{l}\text { Schweizer } \\
\text { Alternativ- } \\
\text { medien Typ } \\
\text { III, IV }\end{array}$ & 8 & 5 & 13 & 0,072 & 79639,029 & 0,001 \\
\hline journal21 & $\begin{array}{l}\text { Schweizer } \\
\text { Alternativ- } \\
\text { medien Typ } \\
\text { III, IV }\end{array}$ & 5 & 0 & 5 & 0,000 & 0,000 & 0,001 \\
\hline burger_ein & $\begin{array}{l}\text { Alternativme- } \\
\text { dien Typ I }\end{array}$ & 1475 & 21 & 1496 & 0,256 & 17001401,108 & 0,283 \\
\hline jouwatch & $\begin{array}{l}\text { Alternativme- } \\
\text { dien Typ I }\end{array}$ & 1257 & 0 & 1257 & 0,000 & 0,000 & 0,245 \\
\hline wach_jetzt_auf & $\begin{array}{l}\text { Alternativme- } \\
\text { dien Typ I }\end{array}$ & 1139 & 14 & 1153 & 0,227 & 1250370,801 & 0,218 \\
\hline deutsch365 & $\begin{array}{l}\text { Alternativme- } \\
\text { dien Typ I }\end{array}$ & 737 & 2 & 739 & 0,571 & 4379,242 & 0,139 \\
\hline wochenblickat & $\begin{array}{l}\text { Alternativme- } \\
\text { dien Typ I }\end{array}$ & 552 & 5 & 557 & 0,200 & 519047,273 & 0,101 \\
\hline Hartes_Geld & $\begin{array}{l}\text { Alternativme- } \\
\text { dien Typ I }\end{array}$ & 526 & 0 & 526 & 0,000 & 0,000 & 0,092 \\
\hline SteinbachErika & $\begin{array}{l}\text { Alternativme- } \\
\text { dien Typ I }\end{array}$ & 499 & 2 & 501 & 0,189 & 256025,847 & 0,093 \\
\hline unzensuriert & $\begin{array}{l}\text { Alternativme- } \\
\text { dien Typ I }\end{array}$ & 442 & 0 & 442 & 0,000 & 0,000 & 0,088 \\
\hline Pressecop24com & $\begin{array}{l}\text { Alternativme- } \\
\text { dien Typ I }\end{array}$ & 380 & 3 & 383 & 1,000 & 17254,902 & 0,083 \\
\hline $\begin{array}{l}\text { COMPACTMa- } \\
\text { gazin }\end{array}$ & $\begin{array}{l}\text { Alternativme- } \\
\text { dien Typ I }\end{array}$ & 283 & 0 & 283 & 0,000 & 0,000 & 0,063 \\
\hline politikstube & $\begin{array}{l}\text { Alternativme- } \\
\text { dien Typ I }\end{array}$ & 259 & 1 & 260 & 1,000 & 19128,000 & 0,056 \\
\hline Zeitgeschehen_ & $\begin{array}{l}\text { Alternativme- } \\
\text { dien Typ I }\end{array}$ & 233 & 36 & 269 & 0,226 & 323256,134 & 0,044 \\
\hline
\end{tabular}




\begin{tabular}{|c|c|c|c|c|c|c|c|}
\hline Account & $\begin{array}{l}\text { Modularity } \\
\text { Class }\end{array}$ & $\begin{array}{c}\text { In- } \\
\text { degree }\end{array}$ & $\begin{array}{c}\text { Out- } \\
\text { degree }\end{array}$ & Degree & $\begin{array}{c}\text { Close- } \\
\text { ness } \\
\text { Centrality }\end{array}$ & $\begin{array}{c}\text { Betweenness } \\
\text { Centrality }\end{array}$ & $\begin{array}{l}\text { Eigen. } \\
\text { Centr. }\end{array}$ \\
\hline AfD_Support & $\begin{array}{l}\text { Alternativme- } \\
\text { dien Typ I }\end{array}$ & 152 & 3 & 155 & 0,142 & 12164,581 & 0,032 \\
\hline TichysEinblick & $\begin{array}{l}\text { «Leucht- } \\
\text { turm»-Alter- } \\
\text { nativmedien } \\
\text { Typ I }\end{array}$ & 3872 & 15 & 3887 & 0,165 & 6548116,563 & 0,696 \\
\hline RolandTichy & $\begin{array}{l}\text { «Leucht- } \\
\text { turm»-Alter- } \\
\text { nativmedien } \\
\text { Typ I }\end{array}$ & 3302 & 17 & 3319 & 0,180 & 5963627,058 & 0,590 \\
\hline cicero_online & $\begin{array}{l}\text { «Leucht- } \\
\text { turm»-Alter- } \\
\text { nativmedien } \\
\text { Typ I }\end{array}$ & 1117 & 25 & 1142 & 0,174 & 2980788,877 & 0,199 \\
\hline Junge_Freiheit & $\begin{array}{l}\text { «Leucht- } \\
\text { turm»-Alter- } \\
\text { nativmedien } \\
\text { Typ I }\end{array}$ & 926 & 0 & 926 & 0,000 & 0,000 & 0,164 \\
\hline KoeppelRoger & $\begin{array}{l}\text { «Leucht- } \\
\text { turm»-Alter- } \\
\text { nativmedien } \\
\text { Typ I }\end{array}$ & 360 & 0 & 360 & 0,000 & 0,000 & 0,066 \\
\hline theeuropean & $\begin{array}{l}\text { «Leucht- } \\
\text { turm»-Alter- } \\
\text { nativmedien } \\
\text { Typ I }\end{array}$ & 71 & 0 & 71 & 0,000 & 0,000 & 0,012 \\
\hline Weltwoche & $\begin{array}{l}\text { «Leucht- } \\
\text { turm»-Alter- } \\
\text { nativmedien } \\
\text { Typ I }\end{array}$ & 65 & 1 & 66 & 0,000 & 0,000 & 0,011 \\
\hline Achgut_com & $\begin{array}{l}\text { «Leucht- } \\
\text { turm»-Alter- } \\
\text { nativmedien } \\
\text { Typ I }\end{array}$ & 42 & 0 & 42 & 0,000 & 0,000 & 0,007 \\
\hline MMnews1 & $\begin{array}{l}\text { «Leucht- } \\
\text { turm»-Alter- } \\
\text { nativmedien } \\
\text { Typ I }\end{array}$ & 37 & 0 & 37 & 0,000 & 0,000 & 0,006 \\
\hline NovoArgumente & $\begin{array}{l}\text { «Leucht- } \\
\text { turm»-Alter- } \\
\text { nativmedien } \\
\text { Typ I }\end{array}$ & 28 & 6 & 34 & 0,181 & 220331,155 & 0,005 \\
\hline RT_Deutsch & $\begin{array}{l}\text { Alternativme- } \\
\text { dien Typ II, III }\end{array}$ & 2156 & 7 & 2163 & 1,000 & 118340,867 & 0,390 \\
\hline jungewelt & $\begin{array}{l}\text { Alternativme- } \\
\text { dien Typ II, III }\end{array}$ & 1023 & 17 & 1040 & 0,600 & 515861,442 & 0,172 \\
\hline
\end{tabular}




\begin{tabular}{|c|c|c|c|c|c|c|c|}
\hline Account & $\begin{array}{l}\text { Modularity } \\
\text { Class }\end{array}$ & $\begin{array}{c}\text { In- } \\
\text { degree }\end{array}$ & $\begin{array}{l}\text { Out- } \\
\text { degree }\end{array}$ & Degree & $\begin{array}{c}\text { Close- } \\
\text { ness } \\
\text { Centrality }\end{array}$ & $\begin{array}{l}\text { Betweenness } \\
\text { Centrality }\end{array}$ & $\begin{array}{l}\text { Eigen. } \\
\text { Centr. }\end{array}$ \\
\hline TeamKenFM & $\begin{array}{l}\text { Alternativme- } \\
\text { dien Typ II, III }\end{array}$ & 559 & 6 & 565 & 0,124 & 1672385,926 & 0,095 \\
\hline de_sputnik & $\begin{array}{l}\text { Alternativme- } \\
\text { dien Typ II, III }\end{array}$ & 336 & 0 & 336 & 0,000 & 0,000 & 0,066 \\
\hline NachDenkSeiten & $\begin{array}{l}\text { Alternativme- } \\
\text { dien Typ II, III }\end{array}$ & 282 & 0 & 282 & 0,000 & 0,000 & 0,049 \\
\hline ddbnews & $\begin{array}{l}\text { Alternativme- } \\
\text { dien Typ II, III }\end{array}$ & 204 & 17 & 221 & 0,221 & 625561,482 & 0,042 \\
\hline cashkurs & $\begin{array}{l}\text { Alternativme- } \\
\text { dien Typ II, III }\end{array}$ & 198 & 0 & 198 & 0,000 & 0,000 & 0,035 \\
\hline DanieleGanser & $\begin{array}{l}\text { Alternativme- } \\
\text { dien Typ II, III }\end{array}$ & 122 & 0 & 122 & 0,000 & 0,000 & 0,020 \\
\hline NewsFrontDE & $\begin{array}{l}\text { Alternativme- } \\
\text { dien Typ II, III }\end{array}$ & 122 & 0 & 122 & 0,000 & 0,000 & 0,022 \\
\hline rotefahnenews & $\begin{array}{l}\text { Alternativme- } \\
\text { dien Typ II, III }\end{array}$ & 89 & 4 & 93 & 0,324 & 40277,316 & 0,014 \\
\hline weltnetzTV & $\begin{array}{l}\text { Alternativme- } \\
\text { dien Typ II, III }\end{array}$ & 76 & 1 & 77 & 1,000 & 126,503 & 0,014 \\
\hline contra_magazin & $\begin{array}{l}\text { Alternativme- } \\
\text { dien Typ II, III }\end{array}$ & 51 & 0 & 51 & 0,000 & 0,000 & 0,011 \\
\hline
\end{tabular}


